\title{
Extended Complex Plane and Riemann Sphere
}

\author{
${ }^{1}$ Egahi, M., ${ }^{2}$ Agbata, B.C., ${ }^{3}$ Ogwuche, O.I., ${ }^{4}$ Soomiyol M. C \\ ${ }^{1,3,4}$ Department of Mathematics/Computer Science, Benue State University, Makurdi, Nigeria \\ ${ }^{2}$ Department of Mathematics, University of Nigeria, Nsukka, Nigeria \\ Corresponding Author: musaegahi@gmail.com +2347037533071 \\ DOI: $10.31364 / S C I R J / v 8 . i 4.2020 . P 0420762$ \\ http://dx.doi.org/10.31364/SCIRJ/v8.i4.2020.P0420762
}

\begin{abstract}
In this paper, we describe vividly the extended complex plane and show how stereographic projection is used to map bijectively points of the extended complex plane to the Riemann Sphere. The chordal metric between points on the sphere is exhaustively treated with particular interest on the image of sums of corresponding points in the plane and on the sphere.
\end{abstract}

Keywords: Complex Plane, Riemann Sphere, infinity, Chordal metric, stereographic projection, Complex analysis.

\section{INTRODUCTION}

Often in complex analysis, we are concerned with functions that become infinite as the variable approaches a given point. It then becomes convenient to include the point at infinity (denoted ' $\infty$ ') in our consideration. The introduction of this point, as an additional point in the complex plane $\mathbb{C}$ turns the complex plane to what we now called the extended complex plane (Conway, 1978). Thus the extended complex plane is $\mathbb{C} \cup\{\infty\} \equiv \mathbb{C}_{\infty}$

Our goal in this paper is to give a concrete picture of this extended complex plane and describe how we can map the points to a unit sphere, which we called the Riemann sphere, using stereographic projection. Our consideration will include when we add two complex numbers on the plane, does it follow for us to just add their corresponding points on the sphere? This is a question that we seek to answer in the paper.

The chordal metric is a metric that measures chordal distance between two points on the sphere, which is an established result (Ahlfors, 1979), we show clear steps on its derivation. This metric is very important because it is used to define other concept in function theory and complex analysis (Blanchard, 1984; Beardon, 1991).

\section{METHODOLOGY}

\section{Stereographic Projection}

We begin by representing $\mathbb{C}_{\infty}$ as a unit sphere in $\mathbb{R}^{3}$. This unit sphere is called the Riemann sphere, $R$. Thus:

$$
R=\left\{\left(x_{1}, x_{2}, x_{3}\right) \in \mathbb{R}^{3}: x_{1}^{2}+x_{2}^{2}+x_{3}^{2}=1\right\}
$$

There is a one-to-one correspondence between the points of $\mathbb{C}_{\infty}$ and $R$, and this correspondence is what we called the stereographic projection. For us to derive the mapping equations we do the following:

Following Egahi (2015), let $N=(0,0,1)$ be the North pole of $R$ and $\mathrm{S}$ be the South - pole, with $S=(0,0,-1)$. Also, we identify $\mathbb{C}$ with $\left\{\left(x_{1}, x_{2}, 0\right): x_{1}, x_{2} \in \mathbb{R}\right\}$ so that $\mathbb{C}$ cuts $R$ along the equator and the positive $x_{3}$ - axis passes through $N$, the north 
pole. Now for each $z$ in $\mathbb{C}_{\infty}$, consider the straight line in $\mathbb{R}^{3}$ through $z$ and $N$. This line intersects the sphere in exactly one point $Z \neq N$. If $|z|<1$, then $Z$ is in the southern hemisphere and if $|z|>1, Z$ is in the northern hemisphere. For $|z|=1, z=Z$. The question now is, what happens to $Z$ as $|z| \rightarrow \infty$ ? Clearly $Z$ approaches $N$, hence we identify $N$ with the point $\infty$ in $\mathbb{C}_{\infty}$. Thus $\mathbb{C}_{\infty}$ is represented as the sphere $R$ (see figure 1 below).

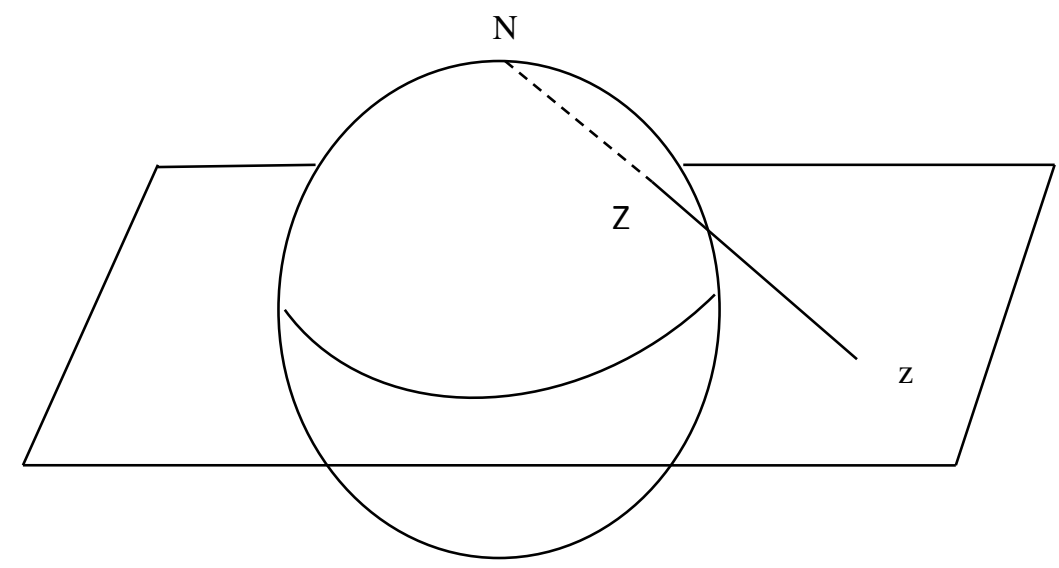

Figure 1: The plane and the sphere intersected together

Now we want to find the formula for the stereographic projection $z=x+i y$ of the point $Z=\left(x_{1}, x_{2}, x_{3}\right)$ on $R$. We will find equations expressing $x_{1}, x_{2}$ and $x_{3}$ in terms of $x$ and $y$. The line in $\mathbb{R}^{3}$ through $z$ and $N$ is given by

$$
\{t N+(1-t) z: t \in \mathbb{R}\}
$$

Since $N=(0,0,1)$ and $z=(x, y, 0)$, we have that $t N+(1-t) z=((1-t) x,(1-t) y, t)$ so that (2) can be written as

$$
\{((1-t) x,(1-t) y, t): t \in \mathbb{R}\}
$$

So that we can find the coordinates of $Z$ if we can find $t$ at which the line intersect $R$. Using (1), we have

$$
\begin{aligned}
1 & =(1-t)^{2} x^{2}+(1-t)^{2} y^{2}+t^{2} \\
1 & =(1-t)^{2}|z|^{2}+t^{2} \\
1-t^{2} & =(1-t)^{2}|z|^{2} \\
t\left(1+|z|^{2}\right) & =|z|^{2}-1 \\
t & =\frac{|z|^{2}-1}{|z|^{2}+1}
\end{aligned}
$$

If we substitute for $t$ in (3), we have that

$$
x_{1}=\frac{2 x}{|z|^{2}+1}, \quad x_{2}=\frac{2 y}{|z|^{2}+1}, \quad x_{3}=\frac{|z|^{2}-1}{|z|^{2}+1}
$$

Writing in terms of $z,(4)$ becomes

$$
x_{1}=\frac{z+\bar{z}}{|z|^{2}+1}, \quad x_{2}=\frac{-i(z-\bar{z})}{|z|^{2}+1}, \quad x_{3}=\frac{|z|^{2}-1}{|z|^{2}+1}
$$


So if $z$ is given in the plane, the corresponding point on the sphere will be as given in (4) or (5). For example $1+i$ on the plane will correspond to $\left(\frac{2}{3}, \frac{2}{3}, \frac{1}{3}\right)$ on the sphere, since $|1+i|^{2}=2$ and $x=1, y=1$. Also $3+2 i$ will correspond to $\left(\frac{3}{7}, \frac{2}{7}, \frac{6}{7}\right)$. These examples buttress our

Next, we want to find $\mathrm{z}$ in the plane if given $\mathrm{Z}$ on $R$. For us to do this, we set $t=x_{3}$ and use (3). Thus $\left(1-x_{3}\right) x=x_{1}$ and $\left(1-x_{3}\right) y=x_{2}$. Then $x=x_{1} /\left(1-x_{3}\right), y=x_{2} /\left(1-x_{3}\right)$ so that

$$
z=x+i y=\frac{x_{1}}{1-x_{3}}+i \frac{x_{2}}{1-x_{3}}=\frac{x_{1}+i x_{2}}{1-x_{3}}
$$

With (6), we can find $z$ that correspond to a given point on the sphere. For example the point $\left(\frac{1}{3}, \frac{2}{3}, \frac{2}{3}\right)$ on the sphere corresponds to $1+2 i$ on the plane. In a similar manner $\left(\frac{2}{3}, \frac{2}{3},-\frac{1}{3}\right)$ correspond to $\frac{1}{2}+\frac{1}{2} i$ and $\left(\frac{1}{2}, \frac{\sqrt{3}}{2}, 0\right)$ correspond to $\frac{1}{2}+\frac{\sqrt{3}}{2} i$. These examples buttress our earlier statement that if $|z|<1$, then $Z$ is in the southern hemisphere and if $|z|>1, Z$ is in the northern hemisphere. Finally, for $|z|=1, z=Z$. We observe that points in the southern hemisphere have their third coordinate value in $\mathbb{R}^{3}$ as negative.

The equations (4) and (6) above relating points of the plane and sphere can also be derived from a geometrical point of view (Needham, 1998). To do this, we use figure 2 below, which is a vertical cross section of figure 1.

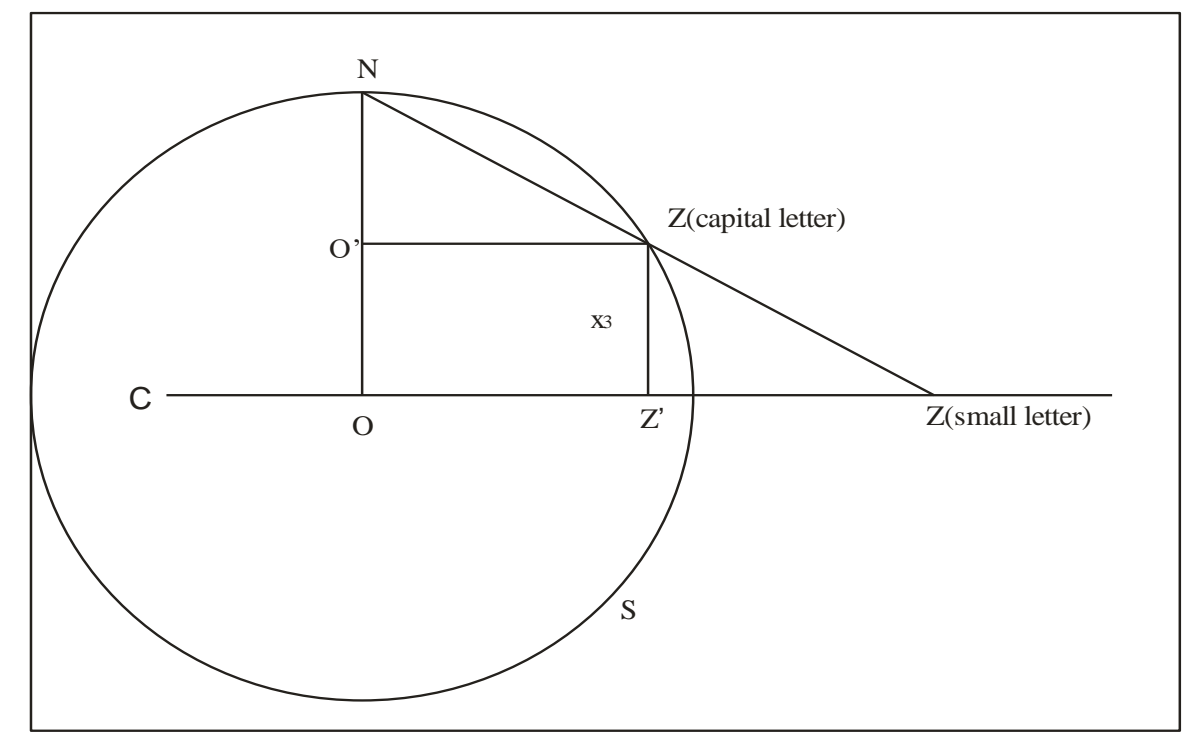

Figure 2: Vertical Cross Section of Figure 1.

Looking at figure $1 z=x+i y$ is the stereographic projection of $Z=\left(x_{1}, x_{2}, x_{3}\right)$ on $R$. Let $z^{\prime}=x_{1}+i x_{2}$ be the foot of the perpendicular from $Z$ to $\mathbb{C}$. Clearly, as seen in the diagram (Figure 2), the desired point $z$ is in the same direction as $z^{\prime}$. To say that two complex numbers in the same direction also mean that they have the same argument. So,

$$
\frac{z}{|z|}=\frac{z^{\prime}}{\left|z^{\prime}\right|}
$$

www.scirj.org

(C) 2020, Scientific Research Journal

http://dx.doi.org/10.31364/SCIRJ/v8.i4.2020.P0420762

This publication is licensed under Creative Commons Attribution CC BY. 
We can rewrite (7) as $z=\frac{|z|}{\left|z^{\prime}\right|} z^{\prime}$. Now from figure 2, triangles $N O^{\prime} Z$ and $N O z$ are similar. As a result, $\frac{|z|}{|z \prime|}=\frac{1}{1-x_{3}}$ from which we obtain

$$
z=\frac{z^{\prime}}{1-x_{3}}=\frac{x_{1}+i x_{2}}{1-x_{3}}
$$

This is the same as (6) above. From (8), we have that

$$
|z|^{2}=\left|\frac{x_{1}+i x_{2}}{1-x_{3}}\right|^{2}=\frac{x_{1}^{2}+x_{2}^{2}}{\left(1-x_{3}\right)^{2}}=\frac{1-x_{3}^{2}}{\left(1-x_{3}\right)^{2}}=\frac{1+x_{3}}{1-x_{3}}
$$

That is $|z|^{2}=\frac{1+x_{3}}{1-x_{3}}$ and solving for $x_{3}$ in (9), we have that $x_{3}=\frac{|z|^{2}-1}{|z|^{2}+1}$. Again from $z=\frac{x_{1}+i x_{2}}{1-x_{3}}$, we have $z\left(1-x_{3}\right)=x_{1}+i x_{2}$. Substituting for $x_{3}$, we find that

$$
x_{1}+i x_{2}=z\left(1-\frac{|z|^{2}-1}{|z|^{2}+1}\right)=z\left(\frac{|z|^{2}+1-|z|^{2}+1}{|z|^{2}+1}\right)=\frac{2 z}{|z|^{2}+1}=\frac{2 x}{|z|^{2}+1}+i \frac{2 y}{|z|^{2}+1}
$$

So we finally have

$$
x_{1}=\frac{2 x}{|z|^{2}+1}, \quad x_{2}=\frac{2 y}{|z|^{2}+1}, \text { and } x_{3}=\frac{|z|^{2}-1}{|z|^{2}+1}
$$

as we have derived in (4) above.

\section{Stereographic Projection of Sums of Complex Number}

In this section we use the method described above to prove that if $Z$ and $Z^{\prime}$ are the stereographic projections of $z$ and $z^{\prime}$ respectively, then $Z+Z^{\prime}$ is not the stereographic projection of $z+z^{\prime}$. To see this we note that $\left(\frac{1}{3}, \frac{2}{3}, \frac{2}{3}\right)$ and $\left(\frac{2}{3}, \frac{1}{3}, \frac{2}{3}\right)$ correspond respectively to $1+2 i$ and $2+i$. Now $\left(\frac{1}{3}, \frac{2}{3}, \frac{2}{3}\right)+\left(\frac{2}{3}, \frac{1}{3}, \frac{2}{3}\right)=\left(1,1, \frac{4}{3}\right)$ does not correspond to $1+2 i+2+i=3+3 i$. In fact $\left(1,1, \frac{4}{3}\right)$ is not even a point on the sphere as $1^{2}+1^{2}+\left(\frac{4}{3}\right)^{2} \neq 1$. Actually, $\left(\frac{6}{19}, \frac{6}{19}, \frac{17}{19}\right)$ is what corresponds to $3+3 i$. We shall state and prove a more general picture to this problem which was posed by Conway (1978).

Problem: Suppose $Z$ and $Z^{\prime}$ are points on the sphere $R$ corresponding to $z$ and $z^{\prime}$ respectively. Let $W$ be the point on the sphere corresponding to $z+z^{\prime}$. Find the coordinates of $W$ in terms of the coordinates of $Z$ and $Z^{\prime}$.

Solution: To solve this problem, we recall that $Z=\left(x_{1}, x_{2}, x_{3}\right)$ correspond to $Z=\frac{x_{1}+i x_{2}}{1-x_{3}}$, and $Z^{\prime}=\left(x_{1}^{\prime}, x_{2}^{\prime}, x_{3}^{\prime}\right)$ correspond to $z^{\prime}=\frac{x_{1}^{\prime}+i x_{2}^{\prime}}{1-x_{3}^{\prime}}$ Now $W=\left(w_{1}, w_{2}, w_{3}\right)$

$$
\begin{aligned}
z+z^{\prime} & =\frac{x_{1}+i x_{2}}{1-x_{3}}+\frac{x_{1}^{\prime}+i x_{2}^{\prime}}{1-x_{3}^{\prime}} \\
& =\frac{\left(1-x_{3}^{\prime}\right)\left(x_{1}+i x_{2}\right)+\left(1-x_{3}\right)\left(x_{1}^{\prime}+i x_{2}^{\prime}\right)}{\left(1-x_{3}\right)\left(1-x_{3}^{\prime}\right)}
\end{aligned}
$$




$$
\begin{aligned}
& =\frac{x_{1}\left(1-x_{3}^{\prime}\right)+x_{1}^{\prime}\left(1-x_{3}\right)+i\left[x_{2}\left(1-x_{3}^{\prime}\right)+x_{2}^{\prime}\left(1-x_{3}\right)\right]}{\left(1-x_{3}\right)\left(1-x_{3}^{\prime}\right)} \\
& =x+i y
\end{aligned}
$$

So that:

$$
x=\frac{x_{1}\left(1-x_{3}^{\prime}\right)+x_{1}^{\prime}\left(1-x_{3}\right)}{\left(1-x_{3}\right)\left(1-x_{3}^{\prime}\right)}, y=\frac{x_{2}\left(1-x_{3}^{\prime}\right)+x_{2}^{\prime}\left(1-x_{3}\right)}{\left(1-x_{3}\right)\left(1-x_{3}^{\prime}\right)}
$$

Now for us to find the coordinates of $W=\left(w_{1}, w_{2}, w_{3}\right)$ in terms of the coordinates of $Z$ and $Z^{\prime}$, we use $w_{1}=\frac{2 x}{|x+i y|^{2}+1}, w_{2}=$ $\frac{2 y}{|x+i y|^{2}+1}, \quad w_{3}=\frac{|x+i y|^{2}-1}{|x+i y|^{2}+1}$, where $x$ and $y$ are as in (11). Hence:

$$
\begin{aligned}
& w_{1}=\frac{2 x}{|x+i y|^{2}+1}=\frac{2 x}{x^{2}+y^{2}+1} \\
& w_{2}=\frac{2 y}{|x+i y|^{2}+1}=\frac{2 y}{x^{2}+y^{2}+1} \\
& w_{3}=\frac{|x+i y|-1}{|x+i y|^{2}+1}=\frac{x^{2}+y^{2}-1}{x^{2}+y^{2}+1}
\end{aligned}
$$

Going back to the illustration before, let $\mathrm{z}=1+2 i, z^{\prime}=2+i$ so that $z+z^{\prime}=3+3 i$. That is $x=3$ and $y=3$, which we can get from

(11) in terms of the coordinates of $Z$ and $Z^{\prime}$. Thus $W=\left(w_{1}, w_{2}, w_{3}\right)=\left(\frac{6}{19}, \frac{6}{19}, \frac{17}{19}\right)$ corresponds to $3+3 i$.

\section{The Chordal Metric}

Here, we define a distance function on the extended plane in the following manner. Let $\mathrm{z}$ and $\mathrm{z}^{\prime}$ belong to $\mathbb{C}_{\infty}$, define the distance from $\mathrm{z}$ to $\mathrm{z}^{\prime}, d\left(z, z^{\prime}\right)$, to be distance between the corresponding points $\mathrm{Z}$ and $\mathrm{Z}^{\prime}$ in $\mathbb{R}^{3}$. If $Z=\left(x_{1}, x_{2}, x_{3}\right)$ and $Z^{\prime}=$ $\left(x_{1}^{\prime}, x_{2}^{\prime}, x_{3}^{\prime}\right)$ then

$$
\begin{gathered}
d\left(z, z^{\prime}\right)=\sqrt{\left(x_{1}-x_{1}^{\prime}\right)^{2}+\left(x_{2}-x_{2}^{\prime}\right)^{2}+\left(x_{3}-x_{3}^{\prime}\right)^{2}} \\
{\left[d\left(z, z^{\prime}\right)\right]^{2}=x_{1}^{2}+x_{2}^{2}+x_{3}^{2}-2\left(x_{1} x_{1}^{\prime}+x_{2} x_{2}^{\prime}+x_{3} x_{3}^{\prime}\right)+x_{1}^{\prime 2}+x_{2}^{\prime 2}+x_{3}^{\prime 2}} \\
=2-2\left(x_{1} x_{1}^{\prime}+x_{2} x_{2}^{\prime}+x_{3} x_{3}^{\prime}\right)\left(\text { as } Z \text { and } Z^{\prime} \text { are on } R\right) .
\end{gathered}
$$

Using (4) we have that

$$
\begin{aligned}
{\left[d\left(z, z^{\prime}\right)\right]^{2}=} & 2-2\left[\left(\frac{2 x}{|z|^{2}+1}\right)\left(\frac{2 x^{\prime}}{\left|z^{\prime}\right|^{2}+1}\right)+\left(\frac{2 y}{|z|^{2}+1}\right)\left(\frac{2 y^{\prime}}{\left|z^{\prime}\right|^{2}+1}\right)+\left(\frac{|z|^{2}-1}{|z|^{2}+1}\right)\left(\frac{\left|z^{\prime}\right|^{2}-1}{\left|z^{\prime}\right|^{2}+1}\right)\right] \\
& =2\left[\frac{\left(|z|^{2}+1\right)\left(\left|z^{\prime}\right|^{2}+1\right)-4 x x^{\prime}-4 y y^{\prime}-\left(|z|^{2}-1\right)\left(\left|z^{\prime}\right|^{2}-1\right)}{\left(|z|^{2}+1\right)\left(\left|z^{\prime}\right|^{2}+1\right)}\right] \\
& =2\left[\frac{2|z|^{2}+2\left|z^{\prime}\right|^{2}-4 x x^{\prime}-4 y y^{\prime}}{\left(|z|^{2}+1\right)\left(\left|z^{\prime}\right|^{2}+1\right)}\right]
\end{aligned}
$$




$$
\begin{aligned}
& =2\left[\frac{2 x^{2}+2 y^{2}+2 x^{\prime 2}+2 y^{\prime 2}-4 x x^{\prime}-4 y y^{\prime}}{\left(|z|^{2}+1\right)\left(\left|z^{\prime}\right|^{2}+1\right)}\right]=2\left[\frac{2\left(x-x^{\prime}\right)^{2}+2\left(y-y^{\prime}\right)^{2}}{\left(|z|^{2}+1\right)\left(\left|z^{\prime}\right|^{2}+1\right)}\right] \\
& =2\left[\frac{2\left|z-z^{\prime}\right|^{2}}{\left(|z|^{2}+1\right)\left(\left|z^{\prime}\right|^{2}+1\right)}\right]=\frac{4\left|z-z^{\prime}\right|^{2}}{\left(|z|^{2}+1\right)\left(\left|z^{\prime}\right|^{2}+1\right)}
\end{aligned}
$$

Hence

$$
d\left(z, z^{\prime}\right)=\frac{2\left|z-z^{\prime}\right|}{\sqrt{\left(|z|^{2}+1\right)\left(\left|z^{\prime}\right|^{2}+1\right)}}
$$

Equation (12) is called the chordal distance or chordal metric.

Now as $0 \in \mathbb{C}_{\infty}$ corresponds to the South Pole $(0,0,-1)$ and $\infty \in \mathbb{C}_{\infty}$ correspond to the North Pole $(0,0,1)$ then $d(0, \infty)$ will be the distance between the South and North Pole. This distance is 2 as $R$ is a unit sphere. So $d(0, \infty)=2$. More generally,

$$
d(z, \infty)=\frac{2}{\sqrt{|z|^{2}+1}}
$$

This is true because $z$ and $\infty$ correspond respectively to $Z=\left(x_{1}, x_{2}, x_{3}\right)$ and $N=(0,0,1)$. Thus $[d(z, \infty)]^{2}=x_{1}^{2}+x_{2}^{2}+$ $\left(1-x_{3}\right)^{2}=2\left(1-x_{3}\right)=\frac{4}{|z|^{2}+1}$, so that $(13)$ holds.

\section{Remark}

We observe that $d(z, w)=d\left(\frac{1}{z}, \frac{1}{w}\right)$. The transformation $z \rightarrow \frac{1}{z}$ correspond to a rotation of $R$ by $\pi$ through $z=1$ and $z=-1$.

\section{Behaviour of Functions at Infinity}

Since the point $\infty$ has been added to $\mathbb{C}$ so that we have $\mathbb{C}_{\infty}$, we use the function $j(z)=1 / z$ to study the point at $\infty$. Using $j$, we take $j(0)=\infty, j(\infty)=0$ and $j: \mathbb{C}_{\infty} \rightarrow \mathbb{C}_{\infty}$ is a bijection. $j(z)$ is well-defined on $\mathbb{C} \backslash\{0\}$. Suppose $f(z)$ is defined on $D \backslash\{\infty\}$, with $D$ a neighbourhood of $\infty$, if we define $f(\infty)=\lim _{z \rightarrow \infty} f(z)$ then $f$ is continuous at $\infty$ and if $f(\infty)=$ $\lim _{z \rightarrow 0}(f o j)(z)$, we say $f$ is analytic at $\infty$ provided $f \circ j$ has corresponding property at 0 .

A function $f(z)$ is analytic at infinity if (i) there exist $N$ a positive integer such that $|z|>N$, (ii) $(f \circ j)(z)$ is analytic at the origin.

Example. Show that $f(z)=\frac{z^{2}+1}{z^{2}-1}$ is analytic at infinity.

Solution. (i) Using the definition, $N=1$. So if $|z|>1, f(z)$ is analytic.

(ii) $(f \circ j)(z)=f(j(z))=f\left(\frac{1}{z}\right)=\frac{1+z^{2}}{1-z^{2}}=1$ if $z=0$. Hence $f\left(\frac{1}{z}\right)$ is analytic at the origin. Therefore the function is analytic at infinity.

Now let $P$ be the point $z=x+i y$ and $P^{\prime}$ be $j(z)=\frac{1}{z}=\frac{x-i y}{|z|^{2}}$. Then $Q=\rho^{-1}(P)$ of $R$ corresponding to $P$ have coordinates $x_{1}=\frac{2 x}{|z|^{2}+1}, \quad x_{2}=\frac{2 y}{|z|^{2}+1}, \quad$ and $x_{3}=\frac{|z|^{2}-1}{|z|^{2}+1}$ in $\mathbb{R}^{3}$ and the coordinates of $Q^{\prime}=\rho^{-1}\left(P^{\prime}\right)$ are: 


$$
\begin{gathered}
x_{1}=\frac{2\left(\frac{x}{|z|^{2}}\right)}{\left|\frac{x-i y}{|z|^{2}}\right|^{2}+1}=\frac{2 x}{|z|^{2}+1}=x_{1} \\
x_{2}=\frac{2\left(\frac{-y}{|z|^{2}}\right)}{\left|\frac{x-i y}{|z|^{2}}\right|^{2}+1}=\frac{-2 y}{|z|^{2}+1}=-x_{2} \\
x_{3}=\frac{\left|\frac{x-i y}{|z|^{2}}\right|^{2}-1}{\left|\frac{x-i y}{|z|^{2}}\right|^{2}+1}=\frac{-\left(|z|^{2}-1\right)}{|z|^{2}+1}=-x_{3}
\end{gathered}
$$

That is $P^{\prime} \equiv j(z)=\frac{1}{z}=\frac{x-i y}{|z|^{2}}$ correspond to $\left(x_{1},-x_{2},-x_{3}\right)$ in $\mathbb{R}^{3}$. Thus $j$ induces the transformation $\rho^{-1} j \rho: Q=\left(x_{1}, x_{2}, x_{3}\right) \rightarrow$ $Q^{\prime}=\left(x_{1},-x_{2},-x_{3}\right)$ of $R$, which is the rotation of $R$ by $180^{\circ}$ about the $x_{1}$ axis. $\rho$ is the stereographic function. This agrees with the remark above.

\section{CONCLUSION}

We have used stereographic projection to map the extended complex plane to the Riemann sphere. The way the plane intersects the sphere makes it possible and clear that a straight line from the North pole to the plane will touch the sphere and the plane in exactly one point. So the point infinity is mapped to the North pole and there is a one to one correspondence between the points of the sphere and plane. The mapping equations are clearly derived, either in matching points from the plane to the sphere or vice versa. Also, we have been able to show how if two complex numbers are added together in the plane, the point corresponding to their sum on the sphere is not just directly adding their various corresponding points on the sphere as the example we used showed.

After showing how stereographic projection is done between the plane and sphere, we now have a complex number system which contain infinity as a number and the distance between any two point on the plane is now viewed as the distance between the corresponding points on the sphere. Hence, this distance is called the chordal distance. Since the longest distance between two points on the sphere is 2 , it is expected distance between any two points on the plane as viewed on the sphere is less or equal to 2. This chordal metric is very useful in defining some concept as we study other types of functions in complex analysis.

\section{REFERENCES}

Ahlfors, L. V (1979) Complex Analysis. 3 edn, USA, McGraw Hill, Inc. 331p

Beardon, A. F. (1991) Iteration of rational functions. Springer, New York, Berlin and Heidelberg 280p

Blanchard, P. (1984). Complex Analytic Dynamics on the Riemann Sphere. Bulletin (New Series) of the American Mathematical Society, 11(1): 85-141 
Conway, J. B. (1978). Function of one Complex Variable. Springer Verlag, New York 322p

Egahi, M (2015). Families of Iterates of Complex Functions. PhD Thesis, University of Jos, Jos Nigeria. 116p

Needham, T. (1998). Visual Complex Analysis, Oxford University Press. USA. First Edition, 592p

www.scirj.org

(C) 2020, Scientific Research Journal

http://dx.doi.org/10.31364/SCIRJ/v8.i4.2020.P0420762

This publication is licensed under Creative Commons Attribution CC BY. 